\title{
Comportamento e bem estar de vacas leiteiras submetidas à secagem: Revisão
}

\author{
$\underline{\text { Ediane Zanin }}^{1}, \underline{\text { Jose Antonio Fregonesi }}^{2}$ Lívia Galiano Mangilli $^{3}$ \\ ${ }^{I}$ Zootecnista, Mestranda em Ciência Animal da Universidade Estadual de Londrina \\ ${ }^{2}$ Médico Veterinário, Docente do Programa de Pós-graduação em Ciência Animal da Universidade Estadual de \\ Londrina, Departamento de Ciências Agrárias \\ ${ }^{3}$ Zootecnista, Doutoranda em Ciência Animal da Universidade Estadual de Londrina \\ *Autor para correspondência, E-mail: ediane.z@hotmail.com
}

\begin{abstract}
RESUMO. O manejo de secagem de vacas realizado cerca de sessenta dias antes da data prevista para o parto é considerado de grande importância para a saúde, bem estar, produção de colostro e leite dos bovinos leiteiros. Apesar de sua importância, ainda existe escassez de informações na literatura científica referente a esse procedimento, mesmo sendo uma prática comum nas fazendas. Mudanças comportamentais como o aumento na frequência de vocalizações, redução no consumo de ração, tempo prolongado de repouso e úbere aparentemente inchado no período de secagem, indicam elevados níveis de estresse e podem ser sinais de desconforto e dor. A frequência e intervalo entre ordenhas e estratégias de alimentação são alternativas disponíveis para manipular a produção de leite e o manejo de secagem de vacas leiteiras. O bem estar de animais de produção se tornou uma grande preocupação para a sociedade, portanto, estratégias de secagem devem ser estudadas. Diante disso, o objetivo do presente trabalho foi realizar uma revisão sobre a importância de um adequado manejo de secagem em relação ao comportamento e bem estar de vacas leiteiras.
\end{abstract}

Palavras chave: fome, lactação, repouso, vocalização.

\section{Behavior and welfare of dairy cows submitted to drying: Review}

\begin{abstract}
Drying of cows carried out about sixty days before the expected date of birthing, is considered of great importance to health, welfare and colostrum and milk production of dairy cattle. Despite it is importance, there is little information in the scientific literature on this procedure, even if it is a common practice on farms. Behavioral changes such as increased frequency vocalizations, reduced feed consumption, prolonged lying and udder apparently swollen during drying period, indicate high levels of stress and can to indicate discomfort and pain. The milking frequency and interval, as well as feed strategies are alternative available to manipulate the milk production and drying of cows. The welfare of farm animals has become a major concern for the society, therefore, drying strategies should be studied. Thus, the objective of this study was to review the importance of an adequate drying management regarding the behavior and welfare of dairy cows.
\end{abstract}

Keywords: hunger, lactation, lying, vocalization.

\section{Introdução}

$\mathrm{O}$ manejo de secagem (interrupção da lactação) realizado cerca de sessenta dias antes da data prevista do parto é considerado de grande importância para a saúde, bem estar, produção de leite e colostro dos bovinos leiteiros. Apesar de sua importância, ainda existe escassez de informações na literatura científica referente a esse procedimento, mesmo sendo um manejo comum nas fazendas leiteiras (Bertulat et al., 2013). O manejo de secagem quando realizado de forma abrupta em vacas de alta produção é caracterizado por causar um desconforto pelo acúmulo e vazamento de leite após a secagem, devido à pressão existente dentro do úbere e interrupção da ordenha (Tucker et al., 2007, Leitner et al., 2007). Porém, enquanto não houver 
quantificação da dor nesses animais, leva-se em consideração o que já está estabelecido, que a dor e o desconforto nesse período são fortes causas do estresse (Martini et al., 2000). Zobel et al. (2013) compararam os efeitos da interrupção abrupta ou gradual da ordenha sobre o vazamento de leite e o comportamento da vaca leiteira. Estes autores demonstraram que as vacas que receberam o tratamento de secagem gradual realizada em cinco dias, permaneceram menos tempo deitadas, aumentaram o tempo despendido na alimentação e diminuíram a produção de leite durante o processo de secagem. Além disso, verificaram que realizando a redução gradual de ordenha em vacas de alta produção, o vazamento de leite depois da secagem foi reduzido.

As mudanças comportamentais, como o aumento na frequência de vocalização, redução no consumo de ração, tempo prolongado de repouso e mudanças fisiológicas, como úbere aparentemente inchado no período de secagem foram associados a elevados níveis de cortisol e podem ser sinais de desconforto e dor. Adicionalmente, o aumento da frequência de vocalização pode estar associado com a fome e a experiência de sofrimento durante o manejo (Valizaheh et al., 2008, Anil et al., 2002).

Diferentes estratégias de alimentação associadas com a frequência de ordenha gradual durante a secagem podem ser utilizadas para tornar esse manejo menos estressante (Odensten et al., 2005). Diante disso, a seguir será feita uma revisão abordando a importância de um manejo adequado de secagem sobre o comportamento e bem estar de vacas leiteiras.

\section{Definindo bem estar animal}

A ciência do bem estar foi criada na década de 1960, porém ainda não existe uma definição única científica sobre o que significa o bem estar animal (Broom \& Molento, 2004). Essa definição única seria importante tanto no estudo como na aplicação de bem estar aos animais em fazendas, laboratórios etc. Após o Governo Britânico criar o Farm Animal Welfare Council - FAWC (Conselho do Bem Estar dos Animais de Fazenda) (FAWC, 2009), que instituiu as cinco liberdades, as quais são aceitas até hoje, como uma descrição geral de bem estar animal.

De acordo com as cinco liberdades os animais devem estar: 1) Livres de fome, sede e desnutrição; 2) Livres de desconforto; 3) Livres de dor, ferimentos e doenças; 4) Livres para expressar seu comportamento; 5) Livres de medo e estresse (FAWC, 2009). O consenso entre pesquisadores e políticos em muitos países sobre as cinco liberdades, é que elas fornecem uma indicação inicial sobre o que deve ser avaliado e disponibilizado aos animais.

Atualmente, o bem estar animal tem sido definido tendo-se como princípios sobre o que é mais desejável ou mais importante para a qualidade de vida dos animais, proporcionando a elaboração de uma vasta metodologia e abordagens para acessar, avaliar e aplicar o bem estar dos animais em fazendas etc. A definição de bem estar animal mais aceita na atualidade é descrita em três conceitos: 1) Saúde e funcionamento animal; 2) Estado afetivo do animal e 3) Adaptações naturais dos animais. As cinco liberdades incorporam os três conceitos, por exemplo, em termos de saúde básica incorporam as doenças estresse físico e lesões, em termos de vida natural incorporam a liberdade para executar comportamento natural, e em termos de estados afetivos incorporam o desconforto, fome, sede, dor, angústia e medo. O bem estar ideal é dado pela associação desses três conceitos (Figura 1, área A); entretanto, em algumas situações eles podem ocorrer dissociados (Fraser, 2009).

\section{Avaliando o bem estar animal}

\section{Comportamento de vacas leiteiras}

Os bovinos são animais gregários, ou seja, que vivem em grupo fazendo circuito diário entre os pastos, cochos de água e ambiente em que são mantidos. Costumam andar em fileira de um lugar para outro e formam hierarquia entre $o$ rebanho, que se associa ao princípio de disputa por espaço e alimento. Quando se sentem ameaçados por predadores, eles se reúnem para buscar segurança no tamanho do grupo ou então utilizam seus chifres como defesa (Grandin \& Deesing, 2014).

No pastejo, a prioridade no acesso a melhor forragem pode firmar a base e estrutura hierárquica de um determinado grupamento de animais. Nos sistemas intensivos o espaço reduzido e constante reagrupamento das vacas provocam aumento da agressividade, porque as vacas têm de competir mais para se alimentar e disputar lugares para deitar. Sob estas condições, alguns indivíduos estarão mais bem sucedidos do que outros no acesso à alimentação ou outros recursos (Galindo et al., 2000). 


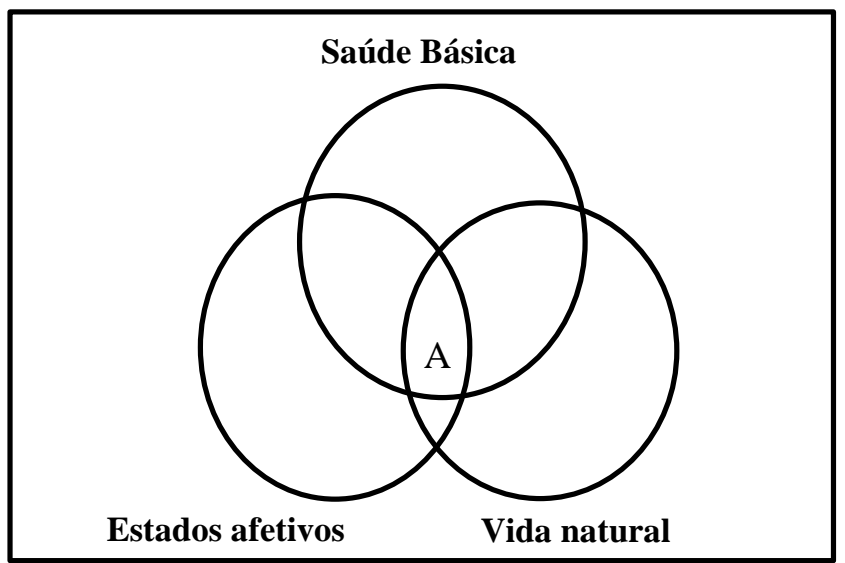

Figura 1. O diagrama mostra os três conceitos de bem estar segundo Fraser (2009). A área "A" representa o bem estar animal ideal por associar os três conceitos.

Estudos realizados com vacas leiteiras indicam que as interações negativas homem vs. animal podem conduzir a interações agonísticas, medo, desregulação hormonal, estresse e dificuldades no manejo, afetando negativamente a produção e o bem estar animal. Ao contrário disso, quando as interações são positivas permitem-se aumentos na produção de leite e melhor desempenho reprodutivo (Rushen et al., 1999a, Breuer et al., 2000).
Os aspectos de comportamento naturais mais importantes para saúde, bem estar e produtividade das vacas, segundo Krawczel \& Grant (2009) são o repouso, alimentação e ruminação. Segundo os autores são necessárias de 12 a 14 horas de repouso e de 3 a 5 horas para a alimentação, estes requisitos constituem de 60 a $80 \%$ das 24 horas do período de atividade (Tabela 1).

Tabela 1. Estimativa do tempo diário para vacas em lactação.

\begin{tabular}{lc} 
Atividade & Tempo dedicado à atividade por dia (hora) \\
\hline Comendo & 3 a 5 (9 a 14 refeições/dia) \\
Deitado/Repouso & 12 a 14 \\
Interação Social & 2 a 3 \\
Ruminando & 7 a 10 \\
Bebendo & 0,5 \\
Atividades de gestão & 2,5 a 3,5 \\
\hline
\end{tabular}

O tempo médio de repouso de vacas e a frequência com que cada animal se deita são influenciados pelo tipo de estabulação, incluindo o tipo de piso, dieta, hierarquia social e estrutura do cubículo, entre outros fatores associados (Dechamps et al., 1989). Fregonesi et al. (2007) avaliaram os efeitos da qualidade da cama sobre o comportamento de deitar vacas leiteiras com acessos a dois tipos de cama (seca ou molhada). No período em que o acesso estava restrito apenas para cama molhada as vacas passaram 8,8 $\pm 0,8 \mathrm{~h} / \mathrm{d}$ deitadas, e quando fornecido a cama seca o tempo aumentou para $13,8 \pm 0,8 \mathrm{~h} / \mathrm{d}$.
Durante o período de livre escolha, todas as vacas passaram mais tempo deitadas na cama seca 12,5 $\pm 0,3 \mathrm{~h} / \mathrm{d}$ do que na cama molhada $0,9 \pm 0,3 \mathrm{~h} / \mathrm{d}$. Os autores concluiram que as vacas leiteiras mostram uma clara preferência por uma superfície seca ao invés de molhada.

O comportamento alimentar é observado pelo tempo e frequência de alimentação, podendo ser influenciado por condições ambientais, manejo e características da dieta, alterando o tempo gasto em cada uma das atividades e sua distribuição ao longo do dia (Marques et al., 2008). O acesso à 
alimentação tem efeito sobre o consumo de matéria seca, pois os animais precisam tempo suficiente para ingerir alimento até atingir o consumo máximo de matéria seca. Se houver limitação ao acesso de alimento menor que 8 horas por dia, a produção de leite pode diminuir até 5\% (NRC, 2001).

$\mathrm{O}$ início da ruminação ocorre entre meia e uma hora e meia após a ingestão do alimento. O número e a duração dos ciclos de ruminação dependem do teor da fibra e tamanho das partículas, número de refeições e quantidade de alimento ingerido. Pode se observar por dia de 4 a 24 períodos de ruminação com 10 a 60 minutos cada, podendo ser gasta 7 horas por dia com ruminação (Silva et al., 2010, Silva et al., 2006, Silva et al., 2007).

O comportamento de vocalização é um aspecto fenotípico do animal que envolve ou não atividades motoras definidas, as quais conduzem ações diárias de sobrevivência e interações sociais (Grandin, 2001, Manteuffel et al., 2004). Como forma de comunicação, os sons são transferidos rapidamente a longas distâncias, em geral servem para manter contato com membros do grupo, ou atrair membros da mesma espécie que estão distantes (Grandin, 1998). Além disso, alguns sons emitidos pelos animais evoluíram como sinais de comunicação de "necessidade," podendo assim considerar vocalizações como indicadores de um estado de bem estar animal (Dawkins, 1998).

\section{Indicadores para avaliar o bem estar de vacas leiteiras}

\section{Indicadores fisiológicos: saúde, doenças e estresse}

A doença é considerada como uma das principais causas que afetam o bem estar de animais de fazenda. Os bovinos de todas as idades e sistemas de produção sofrem com diferentes tipos de doenças endêmicas, infecciosas, não infecciosas e surtos de doenças epidêmicas. Além disso, esses animais sofrem com lesões físicas ocasionadas por deficiências nas instalações ou no manejo (Rushen et al., 2007a). As medidas de saúde são indicadores potencialmente úteis para avaliar o bem estar dos animais (Broom, 2006). Pressupõe-se que, em muitos casos a doença pode estar associada a experiências negativas, como dor, desconforto ou estresse. Em casos de doenças que provocam dores crônicas o sofrimento se estende por longo prazo impactando fortemente $\mathrm{o}$ bem estar (Cerqueira et al., 2012).

Apesar de parecer claro que bem estar animal depende de boas condições de saúde e que medidas da incidência de doenças são usadas para avaliar o bem estar, é necessário estimar o impacto relativo dessas doenças. Não existe uma maneira de medir diretamente o sofrimento causado ao animal pelas diferentes doenças, mas há uma abordagem indireta que consiste em comparar diferentes doenças através da gravidade dos sintomas. A gravidade pode ser avaliada pela duração da doença e probabilidade de morte. Em vacas leiteiras muitas doenças provocam redução na produção de leite e no consumo ração (Rushen et al., 2007b).

O sistema imune quando é ativado utiliza uma quantidade de energia metabólica (Colditz et al., 2012), e muitas doenças ocasionam redução de consumo, assim os recursos metabólicos são limitados e desviados para a função imune, ao invés de produção de leite, crescimento e reprodução. Os parâmetros produtivos podem ser indicadores úteis de bem estar dos animais quando são relacionadas e validadas com fatores conhecidos como dor ou sofrimento, procurando compreender a causa da alteração na produtividade (Rushen et al., 2007b).

A palavra estresse, de origem inglesa, significa pressão ou tensão fisiológica, mental e ou emocional. Portanto, quando um animal está em estado de estresse seu bem estar está afetado. Ao passar dos anos estudos foram desenvolvidos procurando entender o sistema do estresse e assim poder relacioná-lo com o bem estar dos animais (Fraser, 2009).

O modelo representado na figura 2 descreve como o estresse pode afetar o bem estar dos animais. De acordo com Moberg (1996) para obter uma resposta de estresse, ocorre primeiramente o reconhecimento ou a percepção do estressor, em seguida desencadeiam-se respostas comportamentais e fisiológicas, ou seja, as reações de defesa contra o estressor. Se as ações usam muitos recursos biológicos do animal, um estado pré-patológico pode ocorrer, que em casos prolongados resultam em alguma patologia de longa duração. 


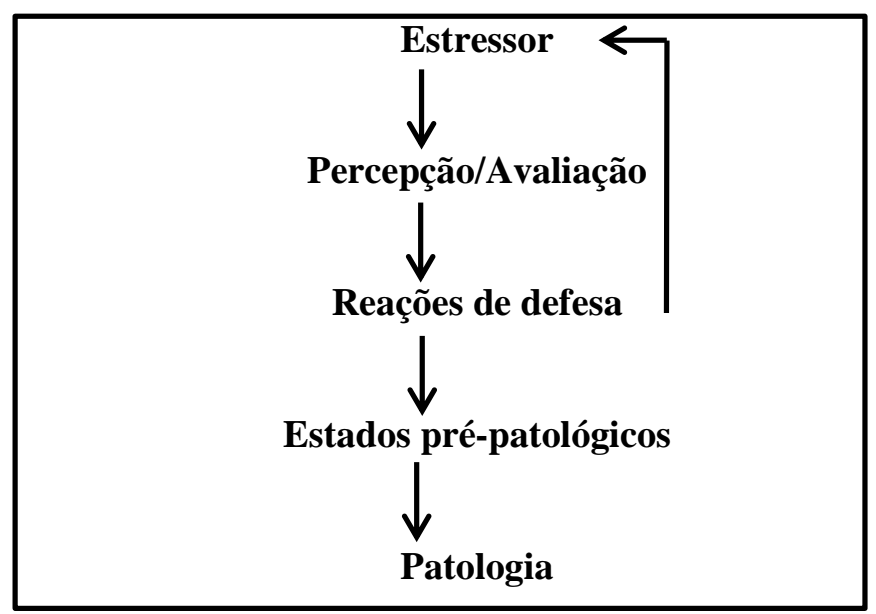

Figura 2. Representação de como estresse afeta o bem estar dos animais Fonte: Moberg (1996)

Indicadores comportamentais: comportamento natural e anormal, aversão, preferência, motivação e vocalização

Um indicador para a avaliação do bem estar animal é pela observação das alterações comportamentais dos animais (Grandin \& Deesing, 2014). Os métodos experimentais de avaliação comportamental, como a observação direta (observador ao vivo) e indireta (auxílio de uso de câmeras com gravações de vídeo e uso de data loggers) têm evoluído nos últimos anos permitindo progressos nas avaliações (Cerqueira et al., 2012).

Pelos procedimentos de aprendizagem e aversão, os animais nos fornecem informações sobre quais tipos de sentimentos e situações eles procuram evitar. Quando um animal é colocado em situações que causam angústia sua resposta comportamental irá depender da natureza da situação à qual está exposto (Rushen et al., $\underline{2007 b}$ ).

Os testes de preferência realizados com animais são utilizados para determinar o que lhes oferece condições de bem estar. Esses testes são usados com bovinos leiteiros para aprimorar determinados tipos de ambientes e preferência por determinados alimentos (Fregonesi et al., 2009, Abade et al., 2015, Tucker et al., 2003). Os testes de preferência apresentam determinadas limitações, por exemplo, o número de opções de escolha fornecidas para os animais nos tratamentos. Nem sempre a opção escolhida pode ser a que o animal prefere, pois pode haver restrições a diversas alternativas de escolha. Além disso, existem diferenças de preferências entre idade, categoria, estados fisiológicos, experiência anterior, entre outros (Rushen et al., 2007b).

O comportamento natural foi amplamente estudado procurando compreender a ligação com o bem estar do animal. Existem comportamentos que os animais necessitam desempenhar a fim de garantir o bem estar. A maioria das espécies, por exemplo, desempenha o comportamento natural de chamadas de alarme para avisar os membros da mesma espécie, que existe predador (Špinka, 2006, Dawkins, 2004). Na espécie bovina, por exemplo, um comportamento natural é afastar-se do rebanho dias antes do parto a procura de um ambiente favorável para parir e oferecer segurança ao filhote (

A grande preocupação na ciência do bem estar animal com relação ao comportamento natural está na incapacidade de realizar determinado comportamento que o animal é motivado. Esse fato pode ser representado quando os animais são alojados em ambientes que impossibilitam desempenhar seu comportamento, como em sistemas de criação intensivo em que as vacas leiteiras permanecem confinadas e não podem se afastar do rebanho antes de parir. A questão referente a essa preocupação é se a interrupção da motivação pode afetar determinado comportamento e se é necessário desempenhá-lo (Rushen et al., 2007b).

Os comportamentos anormais podem indicar que está faltando um elemento importante no ambiente para funcionamento normal do animal e refletir algum estado afetivo negativo. Os bovinos jovens apresentam comportamentos de sugar objetos e urina, lamber e mordiscar, os 
quais em muitos casos são considerados anormais. Um exemplo de comportamento anormal é a atividade de vácuo (enrolar a língua), o qual ocorre em vacas leiteiras adultas que permanecem confinadas (Fraser, 2009).

As vocalizações de animais de criação são produzidas a partir de um conhecimento desenvolvido durante a ontogenia e podem ser moldadas ou não por experiência. Uma determinada vocalização animal pode representar um estado interno do animal (Manteuffel et al., 2004). As reações fisiológicas e/ou comportamentais causadas pelas alterações nesse estado podem fornecer o significado da vocalização (Weary \& Fraser, 1995).

De acordo com Manteuffel et al. (2004) em fazendas leiteiras algumas causas originais de vocalizações podem estar extintas, por exemplo, uma vaca com o úbere cheio vocaliza para atrair sua cria, e a cria por sua vez para atrair a sua alimentação. Outras causas continuam evidentes, como a alteração da frequência de vocalização durante a execução da marca de ferro quente em bezerros (Watts \& Stookey, 1999) e durante a separação de vacas e bezerros (Watts et al., 2001, Marchant-Forde et al., 2002). Também foi demonstrado, segundo Rushen et al. (1999b), que ambientes desconhecidos para vacas leiteiras interferem nos seus padrões sociais e incidem no aumento do comportamento de defecar, urinar e vocalizar.

A tabela 2 apresenta uma síntese dos indicadores comportamentais e fisiológicos utilizados para avaliar o bem estar de bovinos e sua importância para a avaliação.

Tabela 2. Indicadores para avaliação do bem estar de bovinos

\begin{tabular}{|c|c|c|c|}
\hline Tipo do indicador & & Descrição ou exemplos & $\begin{array}{c}\text { Validade/importância do } \\
\text { indicador de BEA }\end{array}$ \\
\hline \multicolumn{4}{|l|}{ Comportamentais } \\
\hline \multirow[t]{4}{*}{$\begin{array}{l}\text { Naturalidade ou } \\
\text { adaptações naturais }\end{array}$} & $\begin{array}{l}\text { Comportamento } \\
\text { natural }\end{array}$ & $\begin{array}{c}\text { Pastar, ruminar, ofegar, repousar } \\
\text { etc. }\end{array}$ & $\begin{array}{c}\text { Depende de evidência } \\
\text { científica }\end{array}$ \\
\hline & $\begin{array}{l}\text { Comportamento } \\
\text { anormal }\end{array}$ & $\begin{array}{l}\text { Automutilação, sugar, mordiscar, } \\
\text { lamber, estereótipos, atividade a } \\
\text { vácuo etc. }\end{array}$ & $\begin{array}{l}\text { Depende de evidência } \\
\text { científica }\end{array}$ \\
\hline & $\begin{array}{l}\text { Preferência Aversão } \\
\text { Motivação }\end{array}$ & $\begin{array}{c}\text { Ambiente ou parte de um } \\
\text { ambiente, manejos, alimentação }\end{array}$ & Direto \\
\hline & Vocalização & $\begin{array}{c}\text { Fome, dor, separação entre } \\
\text { vaca/bezerra, medo, comunicação }\end{array}$ & Direto \\
\hline \multicolumn{4}{|l|}{ Fisiológicos } \\
\hline & Saúde & $\begin{array}{c}\text { Produção e produtividade, } \\
\text { eficiência reprodutiva }\end{array}$ & Indireto \\
\hline & Doença & $\begin{array}{c}\text { Mastites, lesões podais, metrites, } \\
\text { mortalidade, doenças } \\
\text { respiratórias, metabólicas, } \\
\text { reprodutivas }\end{array}$ & Direto \\
\hline & Estresse & $\begin{array}{c}\text { Descorna, separação vaca/bezerro } \\
\text { etc. }\end{array}$ & Indireto \\
\hline
\end{tabular}

Direto: há evidências científicas que afeta o bem estar animal; Indireto: há evidências divergentes que dependem de causa e efeito na saúde ou estado afetivo dos animais. Fonte: o próprio autor

\section{Bem estar de vacas leiteiras submetidas ao processo de secagem}

O bem estar em animais de produção se tornou uma grande preocupação científica e social (Von Keyserlingk et al., 2009). O processo de interrupção da lactação (secagem) das vacas leiteiras, segundo Zobel et al. (2013) é importante para o bem estar e produção na lactação seguinte. Ainda segundo esses autores, o principal problema está relacionado ao aumento de infecções intramamárias, dor e desconforto, devido ao úbere ingurgitado, restrição alimentar e agressividade entre os animais.

De acordo Zobel et al. (2015) poucos trabalhadores do setor de bovinocultura de leite hoje em dia discordariam que as doenças em geral afetam o desempenho do animal e a rentabilidade da fazenda. No entanto, somente o foco na saúde não é suficiente. Preocupações sobre bem estar animal vão além da saúde. 
Conforme Fraser (2009), o bem estar animal é garantido quando os três principais conceitos citados anteriormente são contemplados. $\mathrm{O}$ bem estar de vacas leiteiras durante o manejo de secagem pode ser facilmente avaliado através dos indicadores de dor, fome, saúde básica e adaptações naturais dos animais. Pesquisas na área de bem estar animal têm questionado como os animais respondem ao manejo da interrupção abrupta da ordenha no final da lactação de vacas com alto potencial leiteiro (Zobel et al., 2015).

Uma das experiências que as vacas passam quando não há redução da produção de leite antes da secagem é a dor (Silanikove et al., 2013). A dor pode ocorrer após a interrupção abrupta de ordenha, e está associada com a pressão no úbere, capacidade de armazenamento atingida e aos danos causados nos tecidos da glândula mamária (Bertulat et al., 2013). Porém, o manejo para redução da produção de leite requer redução na alimentação, e essa estratégia pode causar a experiência de fome (Valizaheh et al., 2008).

Está estabelecido que a redução da energia na dieta de vacas antes da secagem reduzirá a produção de leite, mas há poucos trabalhos que avaliam a fome dos animais submetidos a essa alteração na dieta. Essa dieta pode ser composta de alimentos com baixo nível de energia, como o volumoso, ou então o fornecimento reduzido de concentrado. Porém, essa estratégia para diminuir a produção de leite é difícil de ser realizada sem comprometer metabolicamente os animais e sem induzir a fome (Odensten et al., 2007) e (Valizaheh et al., 2008).

A fome é considerada um estado afetivo negativo do animal. As vacas leiteiras durante o manejo de secagem passam por essa experiência, e mesmo sendo em curto prazo pode ter um efeito negativo no bem estar desses animais. Para determinar o estado afetivo são utilizados estudos comportamentais de vocalização (D'Eath et al., 2009). O comportamento de vocalização em vacas adultas pode ser ligado a períodos de sofrimento, isolamento social, dor e fome (Watts \& Stookey, 2000).

Medidas indiretas como a vocalização são utilizadas para avaliar se as vacas leiteiras passam por experiência de dor. Em seu estudo de secagem de vacas com produção de $25 \mathrm{~kg}$ de leite/dia, Silanikove et al. (2013) sugeriu que o aumento de vocalizações era indicativo de úbere cheio de leite ou inchado com ingurgitamento, e causa de dor.
A frustração é um estado afetivo também medido de forma indireta, onde os animais são motivados a adquirir algo pelo oferecimento de uma recompensa. As vacas durante a lactação são motivadas a serem ordenhadas pela recompensa da alimentação concentrada, e quando submetidas ao período de secagem podem passar por um estado de frustração, já que o ciclo de recompensa não é finalizado devido à mudança no manejo (Broom, 2006, Broom \& Molento, 2004). Nessa época as vacas estão mais susceptíveis a infecções na glândula mamária, embora possa ocorrer em qualquer outro período da lactação. Vários estudos têm sido desenvolvidos para prevenção e tratamento dessas infecções, causadas por uma variedade de patógenos contagiosos e ambientais (Halasa et al., 2009).

O fim da lactação nos mamíferos tem como tendência natural ocorrer gradativamente a partir do momento em que a ingestão do leite se faz desnecessária para sua prole (Vitale et al., 1986). Nos dias de hoje a metodologia usada para secagem do leite está muito distante do que ocorre naturalmente, pois no sistema industrializado do setor leiteiro os bezerros são separados das mães logo após o nascimento, e a interrupção da produção de leite é realizada através de diferentes métodos de secagem (Zobel et al., 2015).

\section{Interrupção da lactação - secagem de vacas}

\section{A glândula mamária e as fases da secagem}

$\mathrm{O}$ úbere da vaca possui quatro quartos diferentes e cada um é formado por milhões de alvéolos, os quais são agrupados em lóbulos formando o lobo. O lobo drena o leite para a cisterna da glândula que armazena o leite produzido pelas células secretoras. Em seguida o leite é escoado para a cisterna do teto até ser liberado para o meio externo. A ejeção do leite ocorre pela liberação da ocitocina que atua por sete a dez minutos nas células mioepiteliais que envolvem os alvéolos. Essas quando estimuladas se contraem e comprimem os alvéolos fazendo o leite escoar pelos canais (Beloti, 2015).

As modificações fisiológicas no período de secagem com a interrupção da ordenha são divididas em três fases: a involução ativa da glândula, fase de involução constante e a lactogênese ou colostrogênese. Essas fases apresentam diferenças na função glandular e na atividade das células secretoras da glândula 
mamária, havendo alterações na composição química e celular da secreção no período de secagem (Birgel et al., 2009). O período seco entre as lactações é importante para a regeneração da função produtiva de tecido mamário e preparação para a lactação seguinte.

\section{Estratégias de secagem de vacas}

\section{Frequência e intervalo de ordenha}

A frequência e o intervalo entre as ordenhas podem ser alterados para manipular a produção de leite e a secagem das vacas. A ausência de ordenha para secagem de vacas leiteiras pode ocorrer de duas formas: abrupta ou gradual. A secagem abrupta é realizada em um dia programado, de acordo com a data prevista para o parto ou pela diminuição constante da produção de leite. Na secagem gradual as vacas são submetidas a variações na frequência de ordenha diária e em dias alternados durante uma ou duas semanas antes da secagem definitiva (Birgel et al., 2009).

Em grande parte dos rebanhos comerciais, o manejo de secagem abrupta associado ao uso de antibióticos para prevenção infecções intramamárias é uma preocupação com o bem estar das vacas leiteiras, bem como com o maior risco de infecções na glândula mamária.Bertulat et al. (2013) investigaram o efeito da secagem abrupta sobre os indicadores de estresse em vacas de baixa, média e alta produção, e os efeitos negativos foram apenas em vacas de alta produção. Os efeitos da redução gradual de ordenha contra a interrupção abrupta em vacas leiteiras não são bem compreendidos. Em vacas de alta produção a redução gradual de ordenha resulta em menor vazamento de leite (Zobel et al., 2013).

\section{Restrição alimentar na secagem}

Além do manejo de frequência de ordenha uma das estratégias utilizadas nas fazendas leiteiras é a restrição alimentar das vacas que estão no período de secagem. A redução drástica na quantidade de alimentos para vacas com elevada produção de leite pode ocasionar grave estresse metabólico e induzir problemas de saúde como a mastite (Odensten et al., 2007). Newman et al. (2010) compararam a restrição alimentar com a redução da frequência de ordenha. E observaram redução na a produção de leite antes da secagem $(9,6 \pm 2,9 \mathrm{~kg}$ de leite/d), e facilidade no manejo de secagem com essas duas estratégias adotadas.

Tucker et al. (2009) avaliaram os efeitos de dois procedimentos comuns no manejo de secagem: restrição alimentar $(8$ e $16 \mathrm{~kg}$ de MS/dia) e redução da frequência de ordenha (1 vez e 2 vezes ao dia) sobre as características de comportamento e de úbere antes da secagem e no período seco. As vacas ordenhadas uma vez ao dia com restrição alimentar de $8 \mathrm{~kg}$ de MS/dia, reduziram a produção de leite em $0,95 \mathrm{~kg}(8,9 \pm$ $7 \mathrm{~kg}$ ), apenas $14 \%$ das vacas apresentaram vazamento de leite após dois dias de secagem em comparação com $42 \%$ que recebiam $16 \mathrm{~kg}$ de MS/dia. Além desses benefícios, as vacas passaram menos tempo comendo (7.3 e $8.3 \pm$ $0.28 \mathrm{~h} / \mathrm{d}$ para 8 e $16 \mathrm{~kg} \mathrm{MS} / \mathrm{d}$ ), passaram mais tempo deitadas e vocalizaram mais quando a restrição foi de $8 \mathrm{~kg}$ de MS/dia (0,8 e $0,2 \pm 0,15$ vocalizações/min para 8 e $16 \mathrm{~kg}$ de MS/d). Essa mudança comportamental de vocalização segundo o autor indicou que a restrição alimentar de $8 \mathrm{~kg}$ de $\mathrm{MS} / \mathrm{d}$ pode causar fome, o que seria indesejável para as vacas durante o processo de secagem. Porém, se baseando nas medidas de vazamento de leite, firmeza do úbere e ingestão de matéria seca, a redução da alimentação de até $16 \mathrm{~kg}$ de MS/dia durante o processo de secagem no presente estudo apresentou benefícios para as vacas.

Dentre os procedimentos para secagem a combinação da redução de frequência de ordenha e rações de baixa energia foram avaliadas por Valizaheh et al. (2008) com o objetivo de investigar os efeitos da alimentação com feno de aveia e de capim festuca no declínio da produção de leite e as respostas comportamentais de vacas na secagem. Apesar da redução da produção de leite antes da secagem oferecer benefícios, a restrição alimentar e redução da frequência de ordenha podem causar efeitos sobre o bem estar de vacas leiteiras. O aumento de vocalização neste estudo sugere que com o fornecimento do feno de aveia as vacas podem não ter consumido nutrientes suficientes para garantir a saciedade, assim realizando a sinalização de fome, a qual indica ausência do bem estar (Valizaheh et al., 2008).

\section{Considerações finais}

O manejo de secagem é complexo e há poucos estudos relacionados ao bem estar de vacas durante esse período. A avaliação do 
comportamento animal oferece subsídios para desvendar os diferentes problemas que ocorrem dentro de uma fazenda, laboratório e instituição com os animais de produção. É necessário encontrar uma estratégia de secagem que permita realizá-la de forma adequada sem causar dor, fome e sofrimento para as vacas leiteiras.

\section{Referências Bibliográficas}

Abade, C., Fregonesi, J., Von Keyserlingk, M. \& Weary, D. (2015). Dairy cow preference and usage of an alternative freestall design. Journal of Dairy Science, 98, 960-965.

Anil, S. S., Anil, L. \& Deen, J. (2002). Challenges of pain assessment in domestic animals. Journal of the American Veterinary Medical Association, 220, 313-319.

Beloti, Vanerli. (2015). Leite: obtenção, inspeção e qualidade. Londrina: Editora Planta, 420p.

Bertulat, S., Fischer-Tenhagen, C., Suthar, V., Möstl, E., Isaka, N. \& Heuwieser, W. (2013). Measurement of fecal glucocorticoid metabolites and evaluation of udder characteristics to estimate stress after sudden dry-off in dairy cows with different milk yields. Journal of Dairy Science, 96, 37743787.

Birgel, D., Junior, E. B., Pogliani, F., Raimondo, R., Birgel, E. \& Araújo, W. (2009). Processo de secagem da glâncula mamária de bovinos da raça Holandesa: Avaliação das características microbiológicas da secreção láctea durante o período seco. Arquivos do Instituto Biologico, 76, 517-522.

Breuer, K., Hemsworth, P. H., Barnett, J. L., Matthews, L. R. \& Coleman, G. J. (2000). Behavioural response to humans and the productivity of commercial dairy cows. Applied Animal Behaviour Science, 66, 273288.

Broom, D. M. (2006). Behaviour and welfare in relation to pathology. Applied Animal Behaviour Science, 97, 73-83.

Broom, D. M. \& Molento, C. F. M. (2004). Animal welfare: concept and related issuesreview. Archives of Veterinary Science, 9, 111.

Cerqueira, J. L., Araújo, J. P., Sorensen, J. T. \& Niza-Ribeiro, J. (2012). Alguns indicadores de avaliação de bem-estar em vacas leiteiras.
Revista Portuguesa de Ciencias Veterinarias, 106, 577-580.

Colditz, I. G., Paull, D. R. \& Lee, C. (2012). Social transmission of physiological and behavioural responses to castration in suckling Merino lambs. Applied Animal Behaviour Science, 136, 136-145.

D'Eath, R. B., Tolkamp, B. J., Kyriazakis, I. \& Lawrence, A. B. (2009). 'Freedom from hunger'and preventing obesity: the animal welfare implications of reducing food quantity or quality. Animal Behaviour, 77, 275-288.

Dawkins, M. S. (1998). Evolution and animal welfare. Quarterly Review of Biology, 73, 305-328.

Dawkins, M. S. (2004). Using behaviour to assess animal welfare. Animal Welfare, 13, S3-S8.

Dechamps, P., Nicks, B., Canart, B., Gielen, M. \& Istasse, L. (1989). A note on resting behaviour of cows before and after calving in two different housing systems. Applied Animal Behaviour Science, 23, 99-105.

FAWC. (2009). Farm animal welfare in Great Britain: Past, present and future. Farm Animal Welfare Council, England.

Fraser, D. (2009). Animal behaviour, animal welfare and the scientific study of affect. Applied Animal Behaviour Science, 118, 108117.

Fregonesi, J. A., Veira, D. M., Von Keyserlingk, M. A. G. \& Weary, D. M. (2007). Effects of bedding quality on lying behavior of dairy cows. Journal of Dairy Science, 90, 54685472.

Fregonesi, J. A., von Keyserlingk, M. A. G. \& Weary, D. M. (2009). Cow preference and usage of free stalls compared with an open pack area. Journal of Dairy Science, 92, 54975502.

Galindo, F., Broom, D. M. \& Jackson, P. G. G. (2000). A note on possible link between behaviour and the occurrence of lameness in dairy cows. Applied Animal Behaviour Science, 67, 335-341.

Grandin, T. (1998). The feasibility of using vocalization scoring as an indicator of poor welfare during cattle slaughter. Applied Animal Behaviour Science, 56, 121-128. 
Grandin, T. (2001). Cattle vocalizations are associated with handling and equipment problems at beef slaughter plants. Applied Animal Behaviour Science, 71, 191-201.

Grandin, T. \& Deesing, M. J. (2014). Genetics and behavior during handling, restraint, and herding. In: Grandin, T. \& Deesing, M. J. (eds.) Genetics and the Behavior of Domestic Animals. Second ed. Academic Press, San Diego.

Halasa, T., Østerås, O., Hogeveen, H., Van Werven, T. \& Nielen, M. (2009). Metaanalysis of dry cow management for dairy cattle. Part 1. Protection against new intramammary infections. Journal of Dairy Science, 92, 3134-3149.

Krawczel, P. \& Grant, R. (2009). Effects of cow comfort on milk quality, productivity and behavior. Proceedings of the NMC Annual Meeting, Charlotte, NC, USA.

Leitner, G., Jacoby, S., Maltz, E. \& Silanikove, N. (2007). Casein hydrolyzate intramammary treatment improves the comfort behavior of cows induced into dry-off. Livestock Science, 110, 292-297.

Manteuffel, G., Puppe, B. \& Schön, P. C. (2004). Vocalization of farm animals as a measure of welfare. Applied Animal Behaviour Science, 88, 163-182.

Marchant-Forde, J. N., Marchant-Forde, R. M. \& Weary, D. M. (2002). Responses of dairy cows and calves to each other's vocalisations after early separation. Applied Animal Behaviour Science, 78, 19-28.

Marques, J. A., Pinto, A. P., J.S., A. J. \& Nascimento, W. G. (2008). Intervalo de tempo entre observações para avaliação do comportamento ingestivo de tourinhos em confinamento. Semina: Ciências Agrárias, 29, 955-960.

Martini, L., Lorenzini, R. N., Cinotti, S., Fini, M., Giavaresi, G. \& Giardino, R. (2000). Evaluation of pain and stress levels of animals used in experimental research. Journal of Surgical Research, 88, 114-119.

Moberg, G. P. (1996). Suffering from stress: an approach evaluating the welfare of an animal. Acta Agriculturae Scandinavica, Section A, 46-49.
Newman, K. A., Rajala-Schultz, P. J., DeGraves, F. J. \& Lakritz, J. (2010). Association of milk yield and infection status at dry-off with intramammary infections at subsequent calving. Journal of Dairy Research, 77, 99106.

NRC. (2001). Nutrient Requirements of Dairy Cattle, 7th rev. edn. Natl. Acad. Press, Washington, DC.

Odensten, M. O., Chilliard, Y. \& Holtenius, K. (2005). Effects of two different feeding strategies during dry-off on metabolism in high-yielding dairy cows. Journal of Dairy Science, 88, 2072-2082.

Odensten, M. O., Holtenius, K. \& Waller, K. P. (2007). Effects of two different feeding strategies during dry-off on certain health aspects of dairy cows. Journal of Dairy Science, 90, 898-907.

Rushen, J., de Passillé, A. M. B. \& Munksgaard, L. (1999a). Fear of people by cows and effects on milk yield, behavior, and heart rate at milking. Journal of Dairy Science, 82, 720727.

Rushen, J., Haley, D. \& de Passillé, A. M. (2007a). Effect of softer flooring in tie stalls on resting behavior and leg injuries of lactating cows. Journal of Dairy Science, 90, 3647-3651.

Rushen, J., Passillé, A. M., Von Keyserlingk, M. A. \& Weary, D. M. (2007b). The welfare of cattle. Springer Science \& Business Media.

Rushen, J., Taylor, A. A. \& de Passillé, A. M. 1999b. Domestic animals' fear of humans and its effect on their welfare. Applied Animal Behaviour Science, 65, 285-303.

Silanikove, N., Merin, U., Shapiro, F. \& Leitner, G. (2013). Early mammary gland metabolic and immune responses during natural-like and forceful drying-off in high-yielding dairy cows. Journal of Dairy Science, 96, 64006411.

Silva, R. R., Prado, I. N., Silva, F. F., Almeida, I. C. C., Santana Júnior, H. A., Queiroz, A. C., Carvalho, G. G. P. \& Barroso, D. S. (2010). Comportamento ingestivo diurno de novilhos Nelore recebendo níveis crescentes de suplementação em pastejo de capimbraquiária. Revista Brasileira de Zootecnia, 39, 2073-2080. 
Silva, R. R., Silva, F. F., Prado, I. N., Carvalho, G. G. P., Franco, I. L., Almeida, I. C. C., Cardoso, C. P. \& Ribeiro, M. H. S. (2006). Comportamento ingestivo de bovinos. Aspectos metodológicos. Archivos de Zootecnia, 55, 293-296.

Silva, R. R., Silva, F. F., Prado, I. N., Carvalho, G. G. P., Oliveira, A. P., Chaves, M. A., Mendes, F. B. L., Souza, D. R. \& Pinnheiro, A. A. (2007). Efeito da ração farelada e peletizada sobre o comportamento ingestivo de bezerros Holandeses. Archivos de Zootecnia, 56, 227-238.

Špinka, M. (2006). How important is natural behaviour in animal farming systems? Applied Animal Behaviour Science, 100, 117-128.

Tucker, C., Weary, D. \& Fraser, D. (2003). Effects of three types of free-stall surfaces on preferences and stall usage by dairy cows. Journal of Dairy Science, 86, 521-529.

Tucker, C. B., Dalley, D. E., Burke, J. L. K. \& Clark, D. A. (2007). Milking cows once daily influences behavior and udder firmness at peak and mid lactation. Journal of Dairy Science, 90, 1692-1703.

Tucker, C. B., Lacy-Hulbert, S. J. \& Webster, J. R. (2009). Effect of milking frequency and feeding level before and after dry off on dairy cattle behavior and udder characteristics. Journal of Dairy Science, 92, 3194-3203.

Valizaheh, R., Veira, D. M. \& von Keyserlingk, M. A. G. (2008). Behavioural responses by dairy cows provided two hays of contrasting quality at dry-off. Applied Animal Behaviour Science, 109, 190-200.

Vitale, A. F., Tenucci, M., Papini, M. \& Lovari, S. (1986). Social behaviour of the calves of semi-wild Maremma cattle, Bos primigenius taurus. Applied Animal Behaviour Science, 16, 217-231.

von Keyserlingk, M. A. G., Rushen, J., de Passillé, A. M. \& Weary, D. M. (2009).
Invited review: The welfare of dairy cattleKey concepts and the role of science. Journal of Dairy Science, 92, 4101-4111.

Watts, J. M. \& Stookey, J. M. 1999. Effects of restraint and branding on rates and acoustic parameters of vocalization in beef cattle. Applied Animal Behaviour Science, 62, 125135.

Watts, J. M. \& Stookey, J. M. (2000). Vocal behaviour in cattle: the animal's commentary on its biological processes and welfare. Applied Animal Behaviour Science, 67, 15-33.

Watts, J. M., Stookey, J. M., Schmutz, S. M. \& Waltz, C. S. (2001). Variability in vocal and behavioural responses to visual isolation between full-sibling families of beef calves. Applied Animal Behaviour Science, 70, 255273.

Weary, D. M. \& Fraser, D. (1995). Signalling need: costly signals and animal welfare assessment. Applied Animal Behaviour Science, 44, 159-169.

Zobel, G., Leslie, K., Weary, D. M. \& von Keyserlingk, M. A. G. (2013). Gradual cessation of milking reduces milk leakage and motivation to be milked in dairy cows at dryoff. Journal of Dairy Science, 96, 5064-5071.

Zobel, G., Weary, D. M., Leslie, K. E. \& Von Keyserlingk, M. A. G. (2015). Invited review: Cessation of lactation: Effects on animal welfare. Journal of Dairy Science, 98, 82638277.

\section{Recebido em Fevereiro 1, 2016 Aceito em Março 1, 2016}

License information: This is an open-access article distributed under the terms of the Creative Commons Attribution License, which permits unrestricted use, distribution, and reproduction in any medium, provided the original work is properly cited 